\title{
Pattern of expression of CCN family members Cyr61, CTGF and NOV in human acute and chronic wounds
}

\author{
UZMA MINHAS, TRACEY A. MARTIN, FIONA RUGE, KEITH G. HARDING and WEN G. JIANG \\ Departments of Wound Healing and Surgery, Cardiff University School of Medicine, Cardiff CF14 4XN, UK
}

Received February 2, 2011; Accepted April 4, 2011

DOI: 10.3892/etm.2011.256

\begin{abstract}
The CCN family is a group of extremely cysteinerich proteins that are found within the extracellular matrix and are comprised of cysteine-rich 61 (Cyr61/CCN1), connective tissue growth factor (CTGF/CCN 2) and nephroblastoma overexpressed (NOV/CCN3). Collectively, these proteins stimulate mitosis, adhesion, apoptosis, extracellular matrix production, growth arrest and migration, and regulate angiogenesis, tumour growth, placentation, implantation, embryogenesis and endochondral ossification. Despite such diverse activity, CCN protein function has not been explored in human wounds and healing. In the present study, we investigated the expression of these proteins in samples of normal, acute and chronic wounds using immunohistochemical staining and real-time quantitative RT-PCR. Statistical analysis was performed using the Fisher's exact test. Our results showed that, although all CCN proteins were present in normal, acute and chronic wounds, their expression levels differed, particularly in the case of connective tissue growth factor (CTGF), for which significantly reduced levels were found in chronic wounds compared to acute wounds $(\mathrm{p}<0.002)$. Thus, the lack of CTGF in wound tissues may contribute to the abnormal healing of clinical wounds. This suggests that $\mathrm{CCN}$ proteins may play an important role in human tissue wound healing. This further suggests that human wound healing may be promoted by manipulating the levels of this protein.
\end{abstract}

\section{Introduction}

Originally discovered in the early to mid-1990's, members of the $\mathrm{CCN}$ family are 30 - to $40-\mathrm{kDa}$ proteins that are extremely cysteine-rich (10\% by mass) (1). They are a group of secreted proteins that specifically associate with the extracellular matrix. They are comprised of cysteine-rich 61 (Cyr61/ CCN1), connective tissue growth factor (CTGF/CCN2) and

Correspondence to: Dr Wen G. Jiang, Metastasis and Angiogenesis Research Group, University Department of Surgery, Cardiff University School of Medicine, Cardiff CF14 4XN, UK

E-mail: jiangw@cf.ac.uk

Key words: CCN family proteins, wound healing, acute wounds, chronic wounds nephroblastoma overexpressed (NOV/CCN3). More recently, the Wnt-induced secreted proteins (WISPs) have also been found to belong to the CCN family. Thus, WISP-1 has been named CCN4, WISP-2 is CCN5 and WISP-3 is CCN6 (1-3). Initially it was believed that these proteins were classical growth factors. However, following extensive research we now appreciate that, although $\mathrm{CCN}$ proteins indeed have some independent activity, they principally modify the signalling of other molecules (1). Their function is diverse - they stimulate mitosis, adhesion, apoptosis, extracellular matrix (ECM) production, growth arrest and migration, and regulate angiogenesis, tumour growth, placentation, implantation, embryogenesis and endochondral ossification $(1,2)$. Target cells include fibroblasts, epithelial cells, endothelial cells, smooth muscle cells and neuronal cells. $\mathrm{CCN}$ proteins have a multi-modular mosaic structure, and the diversity in their function is thought to be possibly due to the structural heterogeneity in this modular configuration $(1,2)$.

Despite the increased understanding of $\mathrm{CCN}$ proteins in various biological functions, such as angiogenesis, bone remodelling, cancer and endocrine pathways, to our knowledge their effect on human wounds has not previously been investigated.

The aim of the present study was to assess the expression of CCN family members Cyr61, CTGF and NOV in various wounds, and to investigate whether correlations exist between the CCN family and the nature of wound healing.

\section{Materials and methods}

Materials. Goat anti-human Cyr61, CTGF and NOV antibodies were purchased from Santa Cruz Biotechnology (Santa Cruz, CA, USA). RNA extraction and RT kits were obtained from BioRad (Hemel Hemstead, UK). PCR primers were designed using Beacon Designer (Biosoft International, Palo Alto, CA, USA) and synthesized by Sigma Genesis (Poole, Dorset, UK). Molecular biology-grade agarose and the DNA ladder were obtained from Invitrogen. Mastermix for the routine and quantitative PCR was from Sigma and BioRad, respectively.

Skin biopsies. Fresh frozen skin biopsies were retrieved from the departmental tissue bank and processed as previously reported $(4,5)$. These samples were collected with the approval of the South East Wales Local Research Ethics Committee and stored in accordance to the Human Tissue Act 
Table I. PCR primers used.

\begin{tabular}{ll}
\hline Cyr61 & 5'-GGGCTGGAATGCAACTTC-3' \\
& 5'-AACTGAACCTGACCGTACACGTTTTGGTAGATTCTGGAG-3' \\
& (spanning the third intron; GenBank accession no. AF307860) \\
CTGF & 5'-GAGTGGGTGTGTGACGAG-3' \\
& 5'-ACTGAACCTGACCGTACAGGCAGTTGGCTCTAATCATA-3' \\
& (spanning the fourth intron; NM_001901) \\
NOV & 5'-CTGTGAACAAGAGCCAGAG-3' \\
& 5'-ACTGAACCTGACCGTACACTTGAACTGCAGGTGGAT-3' \\
& (spanning positions 848-849; NM-002514) \\
GAPDH & 5'-CTGAGTACGTCGTGGAGTC-3' \\
& 5'-ACTGAACCTGACCGTACACAGAGATGACCCTTTTG-3'
\end{tabular}

(www.hta.gov.uk). Samples used included normal skin, acute wound tissue and chronic wound tissue.

Normal skin. In order to obtain a comparison with wound tissue, unwounded skin was analysed. Unwounded skin samples were obtained from 10 healthy staff members in our department. Under local anaesthesia, a 3-mm punch biopsy was extracted from the inner region of the upper arm.

Acute wound tissue. Single punch biopsies were obtained from 10 patients with acute wounds after undergoing excision of pilonidal disease. These wounds were judged to be clinically non-infected by a medical wound healing expert from a specialist wound healing unit. These biopsies were obtained from the wound margin incorporating the epidermis and dermis at the wound edge within 6 weeks from the surgical excision.

Chronic wound tissue. Seventeen patients with chronic venous leg ulcers (present for $>6$ months) showing no sign of healing for a duration of 6 weeks were biopsied. Duplex ultrasonography was used to confirm venous disease. These wounds were judged to be clinically non-infected by a medical wound healing expert from a specialist wound healing unit. Again biopsies were obtained from the wound margin incorporating the epidermis and dermis at the wound edge.

Immunohistochemical staining. Immunohistochemical staining was performed using a standard technique employed in our laboratories $(3,4)$. The tissues were frozen-sectioned using a Leica cryostat, at an $8-\mu \mathrm{m}$ thickness. The sections were mounted on Super Frost Plus microscope slides, air dried and then fixed in acetone (Fisher Scientific Ltd., Loughborough, UK) for $15 \mathrm{~min}$. Excess acetone was removed by air drying the sections for $10 \mathrm{~min}$ before being washed three times in Tris-buffered saline (TBS) for 5 min each time. Endogenous peroxidase was blocked by further treating the slides in the blocking solution ( $5 \mathrm{ml}$ of $30 \% \mathrm{H}_{2} \mathrm{O}_{2}$ in $300 \mathrm{ml}$ pure ethanol) for 15 min. Following rehydration, sections were incubated at room temperature with normal blocking solution which contained horse serum (Dako Ltd., High Wycombe, UK). Excess blocking serum was removed, and the sections were probed with the working dilution of primary antibody (produced in 1\% TBS/BSA, dilution 1:150).
Antibody localisation was subsequently identified using a standard streptavidin-biotin peroxidase technique using the Vector Elite ABC kit (Vector Laboratories). This involved incubation with a biotinylated secondary antibody for $30 \mathrm{~min}$, followed by incubation for an additional $30 \mathrm{~min}$ with the avidin-biotin complex reagent provided in the kit. Diaminobenzidine (DAB) $(0.005 \%)$ was then added to the sections, which were incubated in the dark for $5 \mathrm{~min}$. The sections were then rinsed in TBS, followed by tap water and then counterstained with Ehrlrich's haematoxylin solution (BDH-Merck, Poole, UK) for $30 \mathrm{sec}$ and then washed again in tap water for $5 \mathrm{~min}$. The sections were then dehydrated through a graded series of alcohol solutions (BDH-Merck) and mounted in DPX medium (BDH-Merck) under a coverslip.

Positive staining was noted as a brown deposit, and nonstained cells as blue counterstained nucleated cells with no associated brown DAB stain. Images were obtained using a digital camera. Slides were evaluated by two independent researchers.

RNA extraction and complimentary DNA (cDNA) synthesis. Individual biopsies were rapidly thawed and homogenised using the Ultra-Turrax T8 (IKA Labortechnik, Staufen, Germany) in RNA extraction buffer (AbGene). Total cellular RNA was quantified using a spectrophotometer (WPA UV 1101; Biotech Photometer, Cambridge, UK). Reverse transcriptase was performed using $0.5 \mu \mathrm{g}$ of the RNA sample using oligo-dT primer according to the manufacturer's instructions and a reverse transcription kit (Sigma, Poole, Dorset, UK). cDNA was prepared by heating the samples at $47^{\circ} \mathrm{C}$ for $60 \mathrm{~min}$, followed by incubation at $75^{\circ} \mathrm{C}$ for $10 \mathrm{~min}$ to inactivate any reverse transcriptase.

Quantitative analysis of CCN family members. The transcript levels of the CCN family members from the prepared cDNA were determined using a real-time quantitative PCR, based on the Ampliflour ${ }^{\mathrm{TM}}$ technology to determine the expression levels of Cyr61, CTGF, NOV and GAPDH transcripts in acute and chronic wound tissue and in normal skin. The primers used are listed in Table I.

The reaction was carried out using conditions as previously reported (6,7): Hot-Start Q-Mastermix (Abgene), 10 pmol of specific forward primer, 10 pmol of reverse primer which 
A
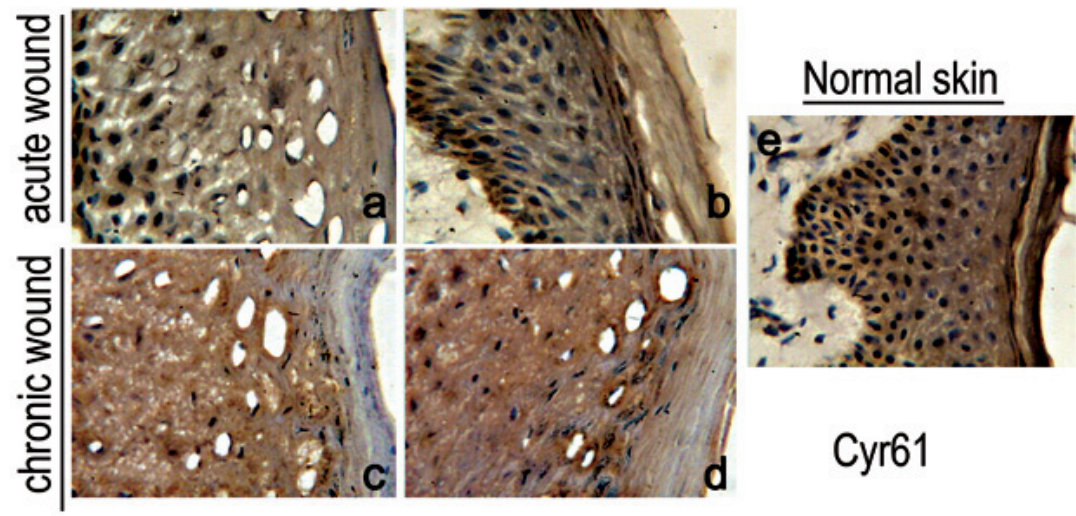

B

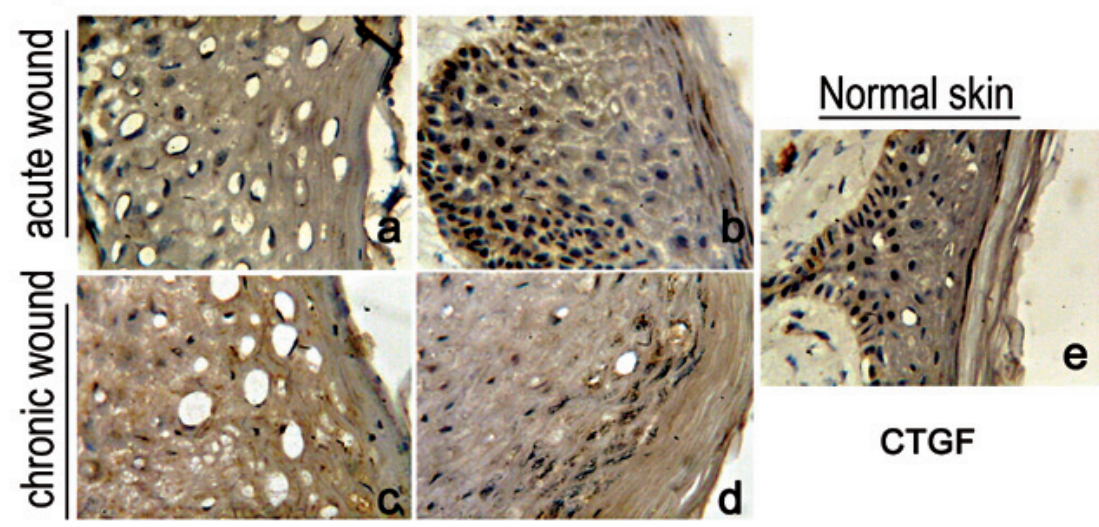

C
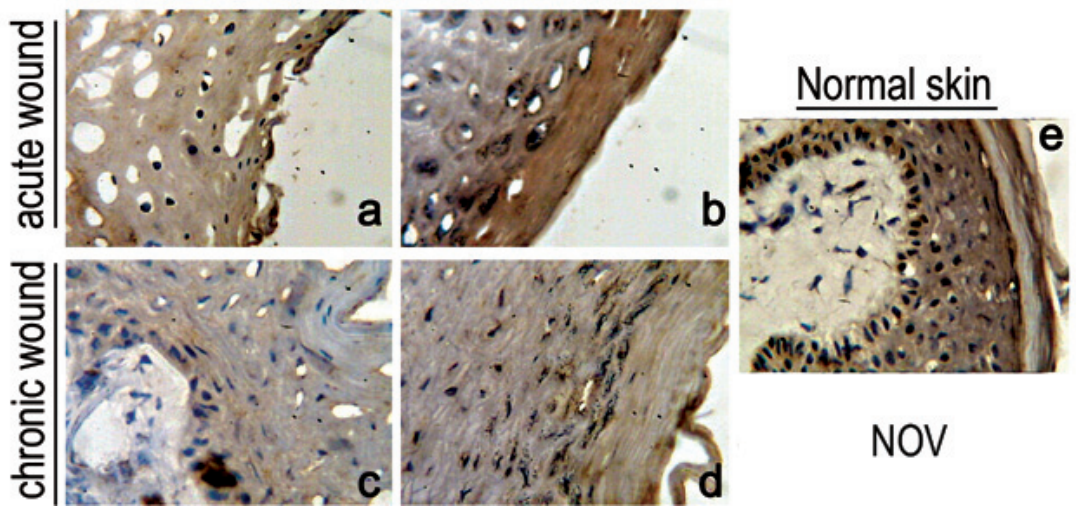

Figure 1. Immunohistochemical staining of Cyr61/CCN1, CTGF/CCN2 and NOV/CCN3. (A) Cyr61 staining: a, acute wound - wound edge; b, acute wound $-2 \mathrm{~mm}$ from wound edge in direction towards normal skin; $\mathrm{c}$, chronic wound - wound edge; $\mathrm{d}$, chronic wound - $2 \mathrm{~mm}$ from wound edge in direction towards normal skin; e, normal skin. (B) CTGF/CCN2 staining: a, acute wound - wound edge; b, acute wound - $2 \mathrm{~mm}$ from wound edge in direction towards normal skin; c, chronic wound - wound edge; d, chronic wound - $2 \mathrm{~mm}$ from wound edge in direction towards normal skin; e, normal skin. (C) NOV/CCN3 staining: a, acute wound - wound edge; b, acute wound - $2 \mathrm{~mm}$ from wound edge in direction towards normal skin; c, chronic wound - wound edge; $\mathrm{d}$, chronic wound $2 \mathrm{~mm}$ from wound edge in direction towards normal skin; e, normal skin.

contains the $\mathrm{Z}$ sequence, 100 pmol of 6 carboxyfluorescein (FAM)-tagged probe (Integren), and cDNA from 50 ng RNA (calculated from the initial RNA in the reverse transcriptase reaction). The reaction was carried out using IcyclerIQ ${ }^{\mathrm{TM}}$ (BioRad) which was equipped with an optic unit that allowed real-time detection of 96 reactions using the following conditions: $94^{\circ} \mathrm{C}$ for $12 \mathrm{~min}, 50$ cycles at $94^{\circ} \mathrm{C}$ for $15 \mathrm{sec}, 55^{\circ} \mathrm{C}$ for $40 \mathrm{sec}$ and $72^{\circ} \mathrm{C}$ for $20 \mathrm{sec}$. The levels of the transcripts were generated from an internal standard (8) that was simultaneously amplified with the samples.

\section{Results}

Protein expression of CCN family members in acute and chronic wound tissues and normal skin tissue. The staining pattern of CCN family members was evaluated according to the spatial distribution. Subdivisions of skin adjacent to the wound edge (WE) and $2 \mathrm{~mm}$ from the wound edge moving towards normal skin were analysed in acute and chronic wounds to allow comparison of the dermis at intervals from the wound bed.

Cyr61. In normal skin, Cyr61 staining was found to be most intense in the dermal layer of the skin. In acute wounds this staining of the dermal layer of the skin was unaltered. However, in chronic wounds the staining in the dermal layer was increased, i.e., was more intensely stained possibly suggesting increased levels of Cyr61 (Fig. 1A).

CTGF. In normal skin, CTGF staining was found to be most intense in the dermal layer of the skin. However, in compar- 
ison to Cyr61, the level of staining was weaker. In acute wounds, the staining of the dermal layer was again weaker and even weaker in chronic wounds possibly suggesting the loss of CTGF (Fig. 1B).

NOV. In normal skin, NOV staining was found to be most intense in the dermal layer of the skin. However, similar to CTGF, the staining in comparison to Cyr61 was weaker. In acute and chronic wounds, staining of the dermal layer of the skin was unaltered, possibly suggesting no change in NOV expression in the wounded states (Fig. 1C).

Analysis of CCN mRNA in normal skin, acute and chronic wound tissues. Both conventional RT-PCR and quantitative real-time RT-PCR were used to analyze the presence and quantity of messages of Cyr61/CCN1, CTGF/CCN2 and NOV/ CCN3. Comparisons were made between acute and chronic wound tissues.

Over $70 \%$ of the chronic wound tissues showed a high level of Cyr61 compared to $40 \%$ of the acute wounds. The difference was nonetheless not statistically significant $(\mathrm{p}=0.13)$ (Table II). The most significant finding was the levels of CTGF/CCN2 in chronic wounds. All (14/14) of the chronic tissues were found to exhibit low levels of the CTGF/CCN2 transcript, compared to only $60 \%$ of acute wounds $(\mathrm{p}=0.0016$; Table III). A trend similar to CTGF/CCN2 was observed for NOV/CCN3; a higher percentage of chronic wound tissues had low levels of the NOV transcript, although this did not reach statistical significance $(\mathrm{p}=0.075$; Table IV).

\section{Discussion}

Non-healing wounds cause great distress to both patients and health professionals. It is estimated that chronic wounds cost the NHS £2.3-3.1 billion/year in the UK (9). Therefore, identifying factors which cause wounds to become chronic may result in the development of possible future therapeutic targets. The present study demonstrated a differential expression of Cyr61/CCN1, CTGF/CCN2 and NOV/CCN3 in acute wound tissue, chronic wound tissue and normal skin particularly for CTGF/CCN2.

The most important finding in the present study was the significant change in $\mathrm{CTGF} / \mathrm{CCN} 2$ expression in chronic wounds. Virtually all of the tissues showed low levels of $\mathrm{CTGF} / \mathrm{CCN} 2$ expression. CTGF/CCN2, also known as insulin-like growth factor binding protein-8 (IGFBP8), was initially identified from endothelial cells and is thought to play a role in connective tissue biology (10). Similar to other IGFBPs, CTGF/CCN2 is known to be involved in the action of IGF (10). Cyr61/CCN1, CTGF/CCN2 and NOV/CCN3 were also found to be induced during tissue repair in adults; however, no comparisons of their expression levels have been evaluated in acute and chronic wound tissues. Elevated levels of CTGF/CCN2 were also found to be a hallmark of fibrosis $(2,10)$. It has been suggested that an inappropriate overexpression of CTGF/CCN2 creates an environment allowing other stimuli to induce potent fibrotic responses (9). It has also been postulated that CTGF/CCN2 is required for maximal adhesive signalling in fibroblasts undergoing active tissue remodelling, such as in embryogenesis, fibrotic cells or tumour cells (10).
Table II. Expression of Cyr61/CCN1 transcript in wound tissues.

\begin{tabular}{lccc}
\hline & $\begin{array}{c}\text { Normal } \\
\text { skin } \\
\mathrm{n}=10\end{array}$ & $\begin{array}{c}\text { Acute wound } \\
\text { tissue } \\
\mathrm{n}=10\end{array}$ & $\begin{array}{c}\text { Chronic wound } \\
\text { tissue } \\
\mathrm{n}=14\end{array}$ \\
\hline High & 4 & 4 & 10 \\
Low & 6 & 6 & 4 \\
\hline
\end{tabular}

Acute wound tissue vs. chronic wound tissue, $\mathrm{p}=0.13$, by Fisher's exact test.

Table III. Expression of CTGF/CCN2 transcript in wound tissues.

\begin{tabular}{lccc}
\hline & $\begin{array}{c}\text { Normal } \\
\text { skin } \\
\mathrm{n}=10\end{array}$ & $\begin{array}{c}\text { Acute wound } \\
\text { tissue } \\
\mathrm{n}=10\end{array}$ & $\begin{array}{c}\text { Chronic wound } \\
\text { tissue } \\
\mathrm{n}=14\end{array}$ \\
\hline High & 7 & 6 & 0 \\
Low & 3 & 4 & 14 \\
\hline
\end{tabular}

Acute wound tissue vs. chronic wound tissue, $p=0.0016$, by Fisher's exact test.

Table IV. Expression of NOV/CCN3 transcript in wound tissues.

\begin{tabular}{lccc}
\hline & $\begin{array}{c}\text { Normal } \\
\text { skin } \\
\mathrm{n}=10\end{array}$ & $\begin{array}{c}\text { Acute wound } \\
\text { tissue } \\
\mathrm{n}=10\end{array}$ & $\begin{array}{c}\text { Chronic wound } \\
\text { tissue } \\
\mathrm{n}=14\end{array}$ \\
\hline High & 1 & 5 & 2 \\
Low & 9 & 5 & 12 \\
\hline
\end{tabular}

Acute wound tissue vs. chronic wound tissue, $\mathrm{p}=0.075$, by Fisher's exact test.

Therefore, it would follow that underexpression of CTGF/ CCN2 creates an environment that impedes wound healing and lack of adhesive signalling in fibroblasts leading to chronicity of wounds as suggested in the present study. This important finding further suggests a therapeutic role of CTGF in chronic wound healing.

Notably, NOV/CCN3 is also present at low levels in chronic wound tissues. Initially discovered from myeloblastosis-associated virus-induced nephroblastoma, the molecule also shares some homology with the IGFBP family (11). Although the functions of NOV/CCN3 are not clear, it has been suggested that the molecule is a keen regulator of haematopoietic stem cells and is associated with tumourigenicity in animal models (12). The result from the present study, although they did not reach statistical significance (due largely to a small sample size), points to a potential role of NOV in abnormal wound healing. This finding warrants further investigation. Although Cyr61/CCN1 has been shown to be strongly linked to cell migration, matrix adhesion and angiogenesis $(1,2)$, one reason to examine this family further is that the present study failed 
to show such a connection. In contrast to our initial hypothesis that this factor may be reduced in chronic wounds, and in clear contrast to CTGF/CCN2 and NOV/CCN3, Cyr61/CCN1 appeared to be present at marginally higher levels in chronic wounds. Although this must be further confirmed in a larger study, it does indicate that Cyr61/CCN1 may have a differential role in the healing process.

In conclusion, the present study demonstrated that CTGF/ CCN2 and NOV/CCN3, to a smaller extent, are aberrantly expressed in wound tissues and are linked to the outcome of clinical wound healing. The findings from the present study indicate that these molecules are potential therapeutic targets in chronic wound healing.

\section{Acknowledgements}

The authors wish to thank Dr Kevin Conway for his assistance in the processing of the tissues.

\section{References}

1. Brigstock DR: The CCN family: a new stimulus package. J Endocrinol 178: 169-175, 2003.

2. Schutze N, Noth U, Schneidereit J, Hendrich C and Jakob F: Differential expression of CCN-family members in primary human bone marrow-derived mesenchymal stem cells during osteogenic, chondrogenic and adipogenic differentiation. Cell Commun Signal 3: 5, 2005.

3. Jiang WG, Watkins G, Fodstad O, Douglas-Jones A, Mokbel K and Mansel RE: Differential expression of the CCN family memebers Cyr61, CTGF and Nov in human breast cancer. Endoc Relat Cancer 11: 781-791, 2004.
4. Conway K, Ruge F, Price P, Harding KG and Jiang WG: Hepatocyte growth factor regulation: an integral part of why wounds become chronic. Wound Repair Reg 15: 683-692, 2007.

5. Jiang WG and Harding KG: Enhancement of wound tissue expansion and angiogenesis by matrix-embedded fibroblast (dermagraft), a role of hepatocyte growth factor/scatter factor. Int J Mol Med 2: 203-210, 1998.

6. Parr C and Jiang WG: Quantitative analysis of lymphangiogenic markers in human colorectal cancer. Int J Oncol 23: 533-539, 2003.

7. Jiang WG, Ye L, Patel $G$ and Harding KG: Expression of WAVEs, the WASP (Wiskott-Aldrich syndrome protein) family of verprolin homologous proteins in human wound tissues and the biological influence on human keratinocytes. Wound Repair Reg 8: 594-604, 2011.

8. Jiang WG, Douglas-Jones A and Mansel RE: Levels of expression of lipoxygenases and cyclooxygenase- 2 in human breast cancer. Prostaglandin, Leukot Essent Fatty Acids 69: 275-281, 2003.

9. Posnett $J$ and Franks PJ: The costs of skin breakdown and ulceration in the UK. In: Skin Breakdown: The Silent Epidemic. Smith \& Nephew Foundation, Hull, pp6-12, 2007.

10. Kim HS, Nagalla SR, Oh Y, Wilson E, Roberts CT and Rosenfeld RG: Identification of a family of low-affinity insulinlike growth factor binding proteins (IGFBPs): characterization of connective tissue growth factor as a member of the IGFBP superfamily. Proc Nat Acad Sci USA 94: 12981-12986, 1997.

11. Doghman M, Arhatte M, Thibout H, et al: Nephroblastoma overexpressed/cysteine-rich protein 61/connective tissue growth factor/nephroblastoma overexpressed gene-3 (NOV/CCN3), a selective adrenocortical cell preapoptotic factor, is downregulated in childhood adrenocortical tumors. J Clin Endocr Metab 92: 3253-3260, 2007.

12. Bork P: The modular architecture of a new family of growth regulators related to connective tissue growth factor. FEBS Lett 327: 125-130, 1993. 